\title{
Una evaluación de las propiedades fisicoquímicas de suelo en sistema productivo de maíz - algodón y arroz en el Valle del Sinú en Colombia
}

\section{An evaluation of soil physicochemical properties in corn - cotton, and rice production systems at the Sinu Valley in Colombia}

\author{
Jose Luis Contreras-Santos ${ }^{1 *}$; Judith Martinez-Atencia ${ }^{2}$; Jorge Cadena-Torres ${ }^{3}$; \\ Rafael-Segundo Novoa-Yanez ${ }^{4}$; Ricardo Tamara-Morelos ${ }^{5}$
}

\begin{abstract}
${ }^{1}$ Ing. Agrícola, Investigador, M.Sc. Corporación Colombiana de Investigación Agropecuaria Agrosavia, C.I. Turipaná. Cereté - Córdoba, Colombia; e-mail: jlcontreras@agrosavia.co; (D) https://orcid.org/0000-0002-8179-3430
\end{abstract}

${ }^{2}$ Ing. Agrícola, Investigador, Ph.D. Corporación Colombiana de Investigación Agropecuaria Agrosavia, C.I. Turipaná. Cereté - Córdoba, Colombia; e-mail: jcmartinez@agrosavia.co; (D) https://orcid.org/0000-0002-8275-2956

${ }^{3}$ Ing. Agrónomo, Investigador, Ph.D. Corporación Colombiana de Investigación Agropecuaria Agrosavia, C.I. Turipaná. Cereté - Córdoba, Colombia; e-mail: jcadena@agrosavia.co; (D) https://orcid.org/0000-0002-5180-2893

${ }^{4}$ Ing. Agrónomo, Investigador, M.Sc. Corporación Colombiana de Investigación Agropecuaria Agrosavia, C.I. Turipaná. Cereté - Córdoba, Colombia; e-mail: rnovoa@agrosavia.co; (D) https://orcid.org/0000-0002-5566-618X

${ }^{5}$ Ing. Agrónomo, Investigador, M.Sc. Corporación Colombiana de Investigación Agropecuaria Agrosavia, C.I. Turipaná. Cereté - Córdoba, Colombia; e-mail: rtamara@agrosavia.co; (D) https://orcid.org/0000-0002-7251-1374

*autor para correspondencia: jlcontreras@agrosavia.co

Cómo citar: Contreras-Santos, J.L.; Martinez-Atencia, J.; Cadena-Torres, J.; Novoa-Yanez, R.S.; Tamara-Morelos, R. 2020. Una evaluación de las propiedades fisicoquímicas de suelo en sistema productivo de maíz - algodón y arroz en el Valle del Sinú en Colombia. Rev. U.D.C.A Act. \& Div. Cient. 23(2):e1375. http://doi.org/10.31910/rudca.v23.n2.2020.1375

Artículo de acceso abierto publicado por Revista U.D.C.A Actualidad \& Divulgación Científica, bajo una licencia Creative Commons CC BY-NC 4.0

Publicación oficial de la Universidad de Ciencias Aplicadas y Ambientales U.D.C.A, Institución de Educación Superior Acreditada de Alta Calidad por el Ministerio de Educación Nacional.

Recibido: Noviembre 3 de 2019 Aceptado: Julio 31 de 2020 Editado por: Ingeborg Zenner de Polanía

\section{RESUMEN}

Los sistemas de producción agrícolas en la subregión Valle del Sinú, en Colombia, en los últimos 50 años, se han basado en la siembra de cultivos en rotación o alternancia de maíz - algodón y arroz, y se caracterizan por el uso intensivo de implementos de mecanización e insumos agrícolas. El objetivo de este estudio fue evaluar las características fisicoquímicas de los suelos, bajo los sistemas productivos de maíz - algodón y arroz e identificar las principales limitantes de suelo, que afectan el desarrollo y la productividad de los cultivos, en la subregión Valle del Sinú. Para lo anterior, se realizaron muestreos de suelo en 64 sitios, distribuidos en cinco municipios del Valle del Sinú, evaluando propiedades físicas y químicas del suelo. El suelo en estudio pertenece al orden Inceptisol, moderadamente profundo, compuesto por arcillas expansivas y horizonte argílico. El suelo, se caracterizó por una reacción acida $(5,82 \pm 0,87$ a $6,78 \pm 0,34)$, contenido medio de MO. La media de la CIC varió en rango, de $18,45 \pm 2,94$ a $22,85 \pm 4,36 \mathrm{cmol}_{(+)} \mathrm{kg}^{-1}$, la CE estuvo no 
salinos, variando de $0,29 \pm 0,09$ a $0,91 \pm 1,70 \mathrm{dS} \mathrm{cm}^{-1}$. Los contenidos de P, S, Ca, Mg y K fueron altos. La densidad aparente mostró valores promedios restrictivos para el desarrollo de raíces en cuatro de los cinco municipios, variando de $1,42 \pm 0,10$ a $1,49 \pm 0,08 \mathrm{~g} \mathrm{~cm}^{-3}$, excepto en San Carlos $\left(1,33 \pm 0,14 \mathrm{~g} \mathrm{~cm}^{-3}\right)$. Los resultados obtenidos evidencian indicios de procesos de degradación de suelo, relacionados con el manejo de los suelos.

Palabras clave: Producción agrícola; Degradación de suelos; Propiedades físico - químicas suelo; Rotación cultivos.

\section{ABSTRACT}

The agricultural production systems in the Valle del Sinú subregion in Colombia, in the last 50 years, have been based on the sowing of crops in rotation and/or alternation of corn - cotton and rice, and are characterized by the intensive use of implements mechanization and agricultural inputs. The aim of this study was to evaluate the physicochemical characteristics of the soils under the corn cotton and rice production systems and identifying the main soil limitations, which affect crop development and productivity, in the Sinú Valley Subregion. For the above, soil sampling was carried out at 64 sites distributed in five municipalities of the Sinú Valley, evaluating the physical and chemical properties of the soil. The soil under study belongs to the order Inceptisol, moderately deep, composed of expansive clays and argillic horizons. The soil was characterized by an acid reaction (5.82 \pm 0.87 to $6.78 \pm 0.34)$, average $\mathrm{MO}$ content. The mean of the CIC varied in the range of $18.45 \pm 2.94$ to $22.85 \pm 4.36 \mathrm{cmol}_{(+)} \mathrm{kg}^{-1}$, the $\mathrm{EC}$ was not saline, varying from $0.29 \pm 0.09$ to $0.91 \pm 1.70 \mathrm{dS} \mathrm{cm}^{-1}$. The contents of $\mathrm{P}, \mathrm{S}$, $\mathrm{Ca}, \mathrm{Mg}$ and $\mathrm{K}$ were high. The apparent density showed restrictive average values for root development in four of the five municipalities, ranging from $1.42 \pm 0.10$ to $1.49 \pm 0.08 \mathrm{~g} \mathrm{~cm}^{-3}$, except in San Carlos $\left(1.33 \pm 0.14 \mathrm{~g} \mathrm{~cm}^{-3}\right)$. The results obtained show evidence of soil degradation processes related to soil management.

Keywords: Agricultural production; Soil degradation; Physical-chemical soil properties; Crop rotation.

\section{INTRODUCCIÓN}

El suelo es un componente fundamental en los sistemas de producción para la nutrición y el soporte de las plantas, convirtiéndose en el elemento esencial para la producción agropecuaria. La pérdida en la calidad del suelo puede conducir a una degradación permanente de la productividad de la tierra (Dutta et al. 2017), asociado a cambios en el uso y las prácticas de manejo de suelo, que afectan su estructura y movilidad o disponibilidad de nutrientes (Abid \& Lal, 2009; Hernández Jiménez et al. 2017).

El crecimiento demográfico y necesidad de producir alimento, en las últimas décadas, ha conllevado a la tecnificación de los sistemas de producción agrícolas y pecuarios, introduciendo grandes volúmenes fertilizantes y uso de maquinaria (laboreo), de forma indiscriminada (Prieto et al. 2010; Castro - Rebolledo et al. 2018), conllevado al deterioro de la calidad y la salud del suelo, lo que ha repercutido en problemas de compactación, disponibilidad de nutrientes y poca o nula actividad de la fauna edáfica (Hernández Jiménez et al. 2017; CEPAL, 2018).

La degradación del suelo, a pesar de estar amenazando la subsistencia de millones de personas en las diferentes regiones, tiende a ser ignorado por las políticas de planificación del sector agropecuario por parte del gobierno y la población en general (Márquez Covarrubias, 2009).

El departamento de Córdoba, en sus últimos 50 años, su vocación ha sido agrícola, con una extensión anual de 83500ha, siendo sus principales cultivos maíz - algodón (alternancia) y arroz (DANE, 2015; FENALCE, 2019). Los métodos agrícolas usados en la producción de estos cultivos, se han concentrado en labranzas intensivas (sobre mecanización), lo que ha generado problemas de compactación en estratos superficiales de suelo (capa arable), induciendo características no deseables (altos densidad aparente, baja porosidad, baja infiltración, mal drenaje, bajo crecimiento de raíces y pérdida de materia orgánica), que afectan el crecimiento, el desarrollo y la productividad de los cultivos (Prieto et al. 2010; Navarro Bravo et al. 2012; Dutta et al. 2017). Estudio realizado por IDEAM \& UDCA (2015), relacionado con el conflicto de uso y la aptitud del suelo, se encontró que el departamento de Córdoba ocupa el tercer puesto en magnitud de suelos degradados.

Los datos relacionados con las propiedades fisicoquímicas de los suelos en la subregión Valle del Sinú, en los sistemas productivos maíz - algodón y arroz son relativamente escasos, más que investigaciones independientes a pequeña escala (parcelas de investigación). Por lo tanto, el objetivo de este estudio fue evaluar las características fisicoquímicas de los suelos, bajo los sistemas productivos de maíz - algodón y arroz, identificando las principales limitantes de suelo, que afectan el desarrollo y la productividad de los cultivos, en la subregión Valle del Sinú.

\section{MATERIALES Y MÉTODOS}

Localización. La presente investigación, se desarrolló en el área dedicada a la producción de cultivos de maíz, algodón y arroz, en la cuenca media y baja del río Sinú, denominada subregión del Valle del Sinú, en el departamento de Córdoba, durante el periodo de transición época seca a lluviosa (abril - mayo) del 2016. Basados en registros de los 10 últimos años de área sembrada y producción de los cultivos de maíz - algodón (alternancia) y arroz, se seleccionaron los municipios de Cereté, San Carlos, Ciénaga de Oro, San Pelayo y Lorica (DANE, 2015; FENALCE, 2019).

En la subregión del Valle del Sinú, se presentan dos periodos climáticos definidos, con un periodo de lluvias, que se inician en mayo y se extienden hasta noviembre, con un rango de precipitación entre 90 a $194 \mathrm{~mm} \mathrm{mes}^{-1}$ y precipitación acumulada de 1.200 a $1.300 \mathrm{~mm}$ año ${ }^{-1}$ y un periodo seco, entre diciembre y marzo, con precipitaciones inferiores a $50 \mathrm{~mm} \mathrm{mes}^{-1}$. La temperatura promedio de esta subregión es de $28^{\circ} \mathrm{C}$, humedad relativa de $82 \%$. Según la clasifi- 
cación de Holdridge (2000), el área de estudio pertenece a la zona agroecológica, denominada Bosque Seco Tropical (Bs'T).

Los suelos característicos del área de estudio corresponden a Fluventic Haplustepts (Lorica, Cereté y Ciénaga de Oro), Aquic Haplustepts (San Pelayo) y Typic Endoaquerts (San Carlos) (USDA, 2014), los cuales presentaron predomino de textura arcillosas, de tipo expansiva (2:1), régimen de humedad údico - acuico y régimen de temperatura isohipertérmico (Tabla 1), con las siguientes características in situ: coloraciones oscuras, profundidad efectiva moderada a profunda, texturas medias a finas, alta presencia de macrofauna (lombrices, hormigas, escarabajos, entre otros), en el primer horizonte de suelo, sin presencia de carbonatos en estratos superficies.

Tabla 1. Descripción de perfiles modales de suelo en cada uno de los municipios evaluados.

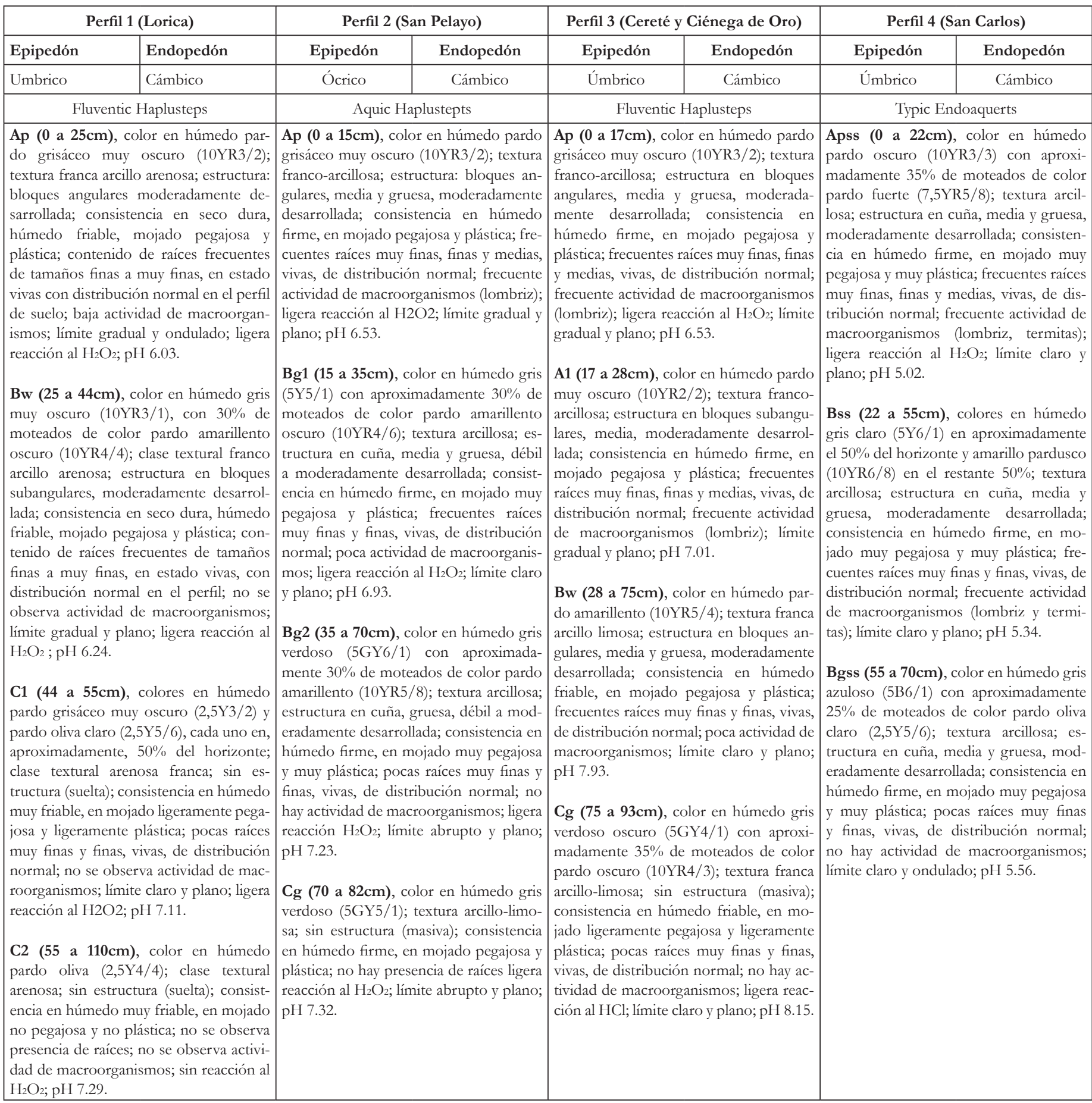


Tamaño muestral. El marco muestral, se identificó con los registros de predios suministrado por FENALCE, UMATAs y asociaciones de productores, encontrando que para el 2016, se contaba con 2.618 predios (Cereté, 913; Ciénaga de Oro, 472; Lorica, 132; San Carlos, 184; San Pelayo, 917), en producción de maíz-algodón y arroz. El tamaño muestral, usando la ecuación de población finita, en estudios cualitativos (Aguilar-Barojas, 2005):

$$
n=\frac{Z^{2} N p q}{p q Z^{2}+(N-1) d^{2}}
$$

Donde: $\mathrm{n}$, tamaño de la muestra; $\mathrm{N}$, tamaño de la población (2.618 predios); Z, Coeficiente para un nivel de confianza del 95\% (1.96); p, Proporción de predios que poseen pérdidas de la capacidad productiva (50\%); q, Proporción de predios que no poseen pérdida de la capacidad productiva (50\%) y d, Error máximo 12,10\%. La fórmula el tamaño muestral (n) arrojó que la población objetivo debía ser de 64 predios, equivalente al 2,44\% de los predios totales registrados.

La distribución de los predios, se realizó teniendo en cuenta los sistemas agrícolas maíz-algodón y arroz: Lorica, se muestrearon 20 predios (10 maíz-algodón y 10 arroz); Cereté, 14 predios (10 maíz-algodón y 4 arroz); Ciénaga de oro, 10 predios (maíz-algodón); San Pelayo, 10 predios (maíz-algodón) y San Carlos, 10 predios (maíz-algodón). Dentro de cada predio, se tomaron muestras por triplicado, bajo un modelo muestral en zig - zag, con distancia entre punto de $50 \mathrm{~m}$, para un total de 192 muestras evaluadas. Los parámetros químicos de suelo, se tomaron, alrededor de cada punto seleccionado, por quintuplicado; luego, estas muestras se homogenizaron, obteniendo $1 \mathrm{~kg}$ de muestra de suelo. El suelo fue muestreado en la profundidad de $0,20 \mathrm{~m}$, colectando muestras disturbadas e indisturbadas, para la evaluación de parámetros fisicoquímicos de suelo.

\section{Variables evaluadas}

Descripción del perfil modal de suelo por municipio. Utilizando la metodología propuesta por IGAC (2017), se realizó descripción de un perfil modal en cada uno de los municipios seleccionados, llegando hasta subgrupo taxonómico y, como forma de corroborar la información obtenida se verificó con el estudio general de suelos y zonificación de tierras, del Departamento de Córdoba (IGAC, 2009).

Propiedades fisicoquímicas del suelo. En el análisis físico del suelo, se determinó: la densidad aparente $(\mathrm{Da})$ : se determinó usando el método del cilindro de volumen conocido $\left(98,17 \mathrm{~cm}^{-3}\right): \mathrm{Da}=$ Mss $/ \mathrm{Vc}$, donde Mss = masa de suelo seco $(\mathrm{g})$ a $105^{\circ} \mathrm{C}$ por $24 \mathrm{hr} \mathrm{y}$ $\mathrm{Vc}=$ volumen del cilindro $\left(\mathrm{cm}^{-3}\right)$; densidad real (Dr) (método del picnómetro); porosidad total $(\mathrm{Pt})$ del suelo, por la relación entre Da y Dr: Pt $=(1-(\mathrm{Da} / \mathrm{Dr})) \times 100$; textura (Bouyoucos); estabilidad de agregados (yoder modificado); macro y microporosidad (curvas de retención de humedad). En el análisis químico, se determinó: concentración de hidrogeniones (potenciómetro, relación 1:1 $\mathrm{P} / \mathrm{V}$ ); acidez y Al intercambiable (KCL 1N); MO (Walkley-Black, combustión en húmedo); fósforo disponible (P, Bray II); potasio (K); calcio (Ca); magnesio (Mg); Sodio $(\mathrm{Na})$ (acetato de amonio 1M a pH 7); azufre (S) y boro (B) (Fosfato monocálcico); elementos menores ( $\mathrm{Cu}, \mathrm{Mn}, \mathrm{Fe}, \mathrm{Zn}$; Olsen modificado) y conductividad eléctrica (conductímetro). Para la determinación de las propiedades fisicoquímicas, se siguió la metodología propuesta por IGAC (2006).

Análisis estadísticos. Inicialmente, se realizó un análisis descriptivo de los parámetros fisicoquímicos de suelo. Para el caso, de las propiedades químicos de suelo, se describieron mediante análisis de frecuencia de rango de la concentración de nutrientes (ICA, 1992). Adicional, se realizó un análisis de variancia; en los casos que se detectaron diferencias significativas $(\mathrm{p}=0,05)$, se realizó prueba de comparación de medias HSD de Tukey, con el fin de comparar el comportamiento de las propiedades fisicoquímicas entre municipios; asimismo, se realizaron análisis de correlación lineal simple de Pearson y análisis de componentes principales (ACP), mediante procedimiento PROC FACTOR, para seleccionar un conjunto mínimo de datos (CMD), basado en un enfoque estadístico, según el procedimiento descrito por Gómez \& Gómez (1984).

\section{RESULTADOS Y DISCUSIÓN}

Los resultados obtenidos en este estudio, así como las discusiones, se dividieron en dos incisos.

Propiedades físicas del suelo. La densidad aparente $(\mathrm{Da}, \mathrm{p}>0,05)$ mostró valores elevados en todos los municipios, que oscilaron,

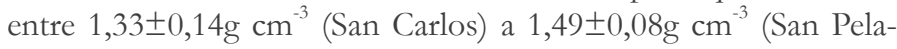
yo), con predomino de textura franco-arcillo-arenoso (Tabla 2). De acuerdo con Burt (2014), valores superiores a $1,40 \mathrm{~g} \mathrm{~cm}^{-3}$, asociados a este grupo textural, son valores restrictivos para el crecimiento de las raíces. La porosidad total $(\mathrm{Pt})$ del suelo en los municipios muestreados fue inferior al 50\% (Tabla 2). En su estudio, Fu et al. (2019) informaron que, valores inferiores al $50 \%$ en la porosidad total del suelo, se relacionan con procesos de compactación (poca o nula infiltración del agua, baja difusión de oxígeno y reduciendo el tamaño de poros estructurales) y baja disponibilidad de nutrimentos en el suelo (Hernández Jiménez et al. 2017). Porosidad total mostró correlacion significativa positiva con $\mathrm{MO}$ y Cu e inversa con el Fe $(\mathrm{r}=$ -0,38). Valores altos de $\mathrm{MO}$ y $\mathrm{Cu}$ favorecen la Pt. Alta presencia de óxidos de hierro en el suelo aumentan los procesos de cementación (Duiker et al. 2003).

Propiedades químicas de suelo. El análisis (descriptivo) de los resultados mostró comportamiento diferencia significativo $(\mathrm{p}<0,05)$ respecto a la reacción del suelo $(\mathrm{pH}), \mathrm{Ca}$ intercambiable, elementos menores ( $\mathrm{Fe}, \mathrm{Cu}, \mathrm{Zn}$ y B) y las relaciones catiónicas, en los municipios evaluados (Tabla 3).

La reacción del suelo $(\mathrm{pH})$ fue mayor en el municipio de San Carlos $(5,82 \pm 0,87)$, valor que indica acidez en el suelo. En los otros municipios, el rango de variación del $\mathrm{pH}$ fue $6,39 \pm 0,47$ a $6,78 \pm 0,34$ (ligeramente ácido a neutro). Igualmente, mediante análisis de frecuencia (Figura 1A), se observó el pH del suelo superficial, bajo los cultivos de maíz - algodón y arroz (inundable); dentro de los mu- 
Tabla 2. Características físicas del suelo en la zona de estudio (Valle del Sinú, departamento de Córdoba, Colombia).

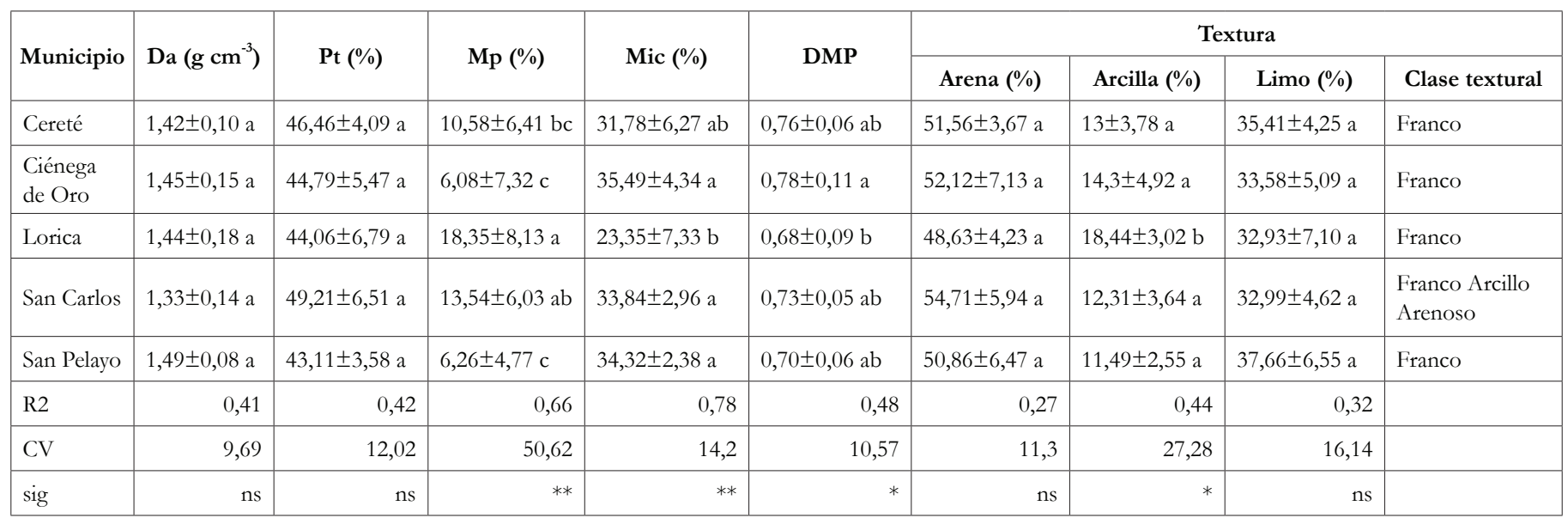

Da: densidad aparente $\left(\mathrm{g} \mathrm{cm}^{-3}\right)$; Pt: porosidad total del suelo; Mp: macro porosidad; Mic: Microporosidad todas estas variables expresadas en porcentaje (\%); DMP: diámetro medio ponderado expresado en milímetros (mm); sig: significancia estadística; ns: no significativo ( $p>0,05)$; * significativo $(\mathrm{p}<0,05)$; ${ }^{* *}$ altamente significativo ( $\left.<<0,0001\right)$; R2: coeficiente de determinación; CV: coeficiente de variación.

Tabla 3. Características químicas de los suelos en la zona de estudio (Valle del Sinú, departamento de Córdoba, Colombia).

\begin{tabular}{|c|c|c|c|c|c|c|c|c|c|c|c|c|c|}
\hline \multirow{2}{*}{$\begin{array}{c}\text { MUNICIPIO } \\
\text { Observaciones }\end{array}$} & \multicolumn{2}{|l|}{ Cereté } & \multicolumn{2}{|c|}{ Ciénaga de Oro } & \multicolumn{2}{|l|}{ Lorica } & \multicolumn{2}{|l|}{ San Carlos } & \multicolumn{2}{|l|}{ San Pelayo } & \multirow{2}{*}{\multicolumn{3}{|c|}{ ANOVA }} \\
\hline & \multicolumn{2}{|l|}{14} & \multicolumn{2}{|l|}{10} & \multicolumn{2}{|l|}{20} & \multicolumn{2}{|l|}{10} & \multicolumn{2}{|l|}{10} & & & \\
\hline \multicolumn{11}{|l|}{ Propiedades } & $\mathrm{R} 2$ & $\mathrm{CV}$ & sig \\
\hline $\mathrm{pH}$ & $6,77 \pm 0,44$ a & $\mathrm{N}$ & $6,55 \pm 0,93$ a & LA & $6,39 \pm 0,47 \mathrm{ab}$ & LA & $5,82 \pm 0,87 \mathrm{~b}$ & Ac & $6,78 \pm 0,34$ a & $\mathrm{N}$ & 0,53 & 8,74 & $*$ \\
\hline $\mathrm{MO}(\%)$ & $2,72 \pm 0,81 \mathrm{a}$ & M & $2,57 \pm 0,97 \mathrm{a}$ & M & $2,05 \pm 0,68 \mathrm{a}$ & M & $2,66 \pm 1,31$ a & M & $2,11 \pm 0,36 \mathrm{a}$ & M & 0,36 & 36,09 & ns \\
\hline $\mathrm{CE}(\mathrm{dS} \mathrm{cm}-1)$ & $0,52 \pm 0,48$ a & NS & $0,49 \pm 0,19 \mathrm{a}$ & NS & $0,91 \pm 1,70 \mathrm{a}$ & NS & $0,38 \pm 0,12 \mathrm{a}$ & NS & $0,29 \pm 0,09$ a & NS & 0,33 & 174,66 & $\mathrm{~ns}$ \\
\hline $\mathrm{CIC}(\mathrm{cmol}(+) \mathrm{kg}-1)$ & $22,85 \pm 4,36 \mathrm{a}$ & A & $22,96 \pm 2,97 \mathrm{a}$ & A & $20,72 \pm 7,44$ a & A & $18,45 \pm 2,94 \mathrm{a}$ & B & $21,08 \pm 2,74 \mathrm{a}$ & A & 0,49 & 21,7 & $\mathrm{~ns}$ \\
\hline \multicolumn{14}{|l|}{ Elementos Mayores } \\
\hline S (mg kg-1) & $21,68 \pm 41,19 \mathrm{a}$ & A & $17,03 \pm 17,08 \mathrm{a}$ & A & $32,92 \pm 49,47 \mathrm{a}$ & A & $19,89 \pm 10,99 \mathrm{a}$ & A & $8,05 \pm 2,36$ a & B & 0,29 & 169,79 & $\mathrm{~ns}$ \\
\hline $\mathrm{P}(\mathrm{mg} \mathrm{kg}-1)$ & $34,18 \pm 31,38 \mathrm{a}$ & M & $33,82 \pm 58,81$ a & M & $8,20 \pm 5,59 \mathrm{a}$ & B & $14,44 \pm 20,26$ a & B & $21,73 \pm 17,39 \mathrm{a}$ & M & 0,44 & 133,93 & ns \\
\hline $\mathrm{Ca}(\mathrm{cmol}(+) \mathrm{kg}-1)$ & $13,68 \pm 11,06$ a & A & $12,88 \pm 4,42 \mathrm{a}$ & A & $12,43 \pm 5,23 \mathrm{ab}$ & A & $9,43 \pm 4,34 \mathrm{~b}$ & A & $12,93 \pm 4,69 \mathrm{a}$ & A & 0,54 & 20,84 & ** \\
\hline $\mathrm{Mg}(\mathrm{cmol}(+) \mathrm{kg}-1)$ & $8,13 \pm 2,34$ a & A & $8,65 \pm 2,00 \mathrm{a}$ & A & $7,49 \pm 3,08$ a & A & $7,59 \pm 1,83$ a & A & $7,43 \pm 1,22$ a & A & 0,37 & 29,94 & ns \\
\hline $\mathrm{K}(\mathrm{cmol}(+) \mathrm{kg}-1)$ & $0,88 \pm 0,59 \mathrm{a}$ & A & $0,83 \pm 1,41 \mathrm{a}$ & A & $0,35 \pm 0,22$ a & M & $0,79 \pm 0,72 \mathrm{a}$ & A & $0,62 \pm 0,23$ a & A & 0,49 & 86,85 & ns \\
\hline $\mathrm{Na}(\mathrm{cmol}(+) \mathrm{kg}-1)$ & $0,14 \pm 0,07 \mathrm{a}$ & B & $0,16 \pm 0,17 \mathrm{a}$ & B & $0,42 \pm 0,59 \mathrm{a}$ & B & $0,18 \pm 0,22 \mathrm{a}$ & B & $0,11 \pm 0,02 \mathrm{a}$ & B & 0,36 & 159,34 & $\mathrm{~ns}$ \\
\hline \multicolumn{14}{|l|}{ Elementos Menores } \\
\hline $\mathrm{Fe}(\mathrm{mg} \mathrm{kg}-1)$ & $70,76 \pm 35,55 \mathrm{~b}$ & A & $\begin{array}{l}101,82 \pm 139,31 \\
\mathrm{~b}\end{array}$ & A & $90,80 \pm 64,77 \mathrm{~b}$ & A & $443,37 \pm 531,49 \mathrm{a}$ & & $71,17 \pm 48,19$ & & 0,5 & 154,78 & ** \\
\hline $\mathrm{Cu}(\mathrm{mg} \mathrm{kg}-1)$ & $5,17 \pm 1,43 \mathrm{~b}$ & A & $4,57 \pm 2,97 \mathrm{~b}$ & A & $4,89 \pm 2,95 \mathrm{~b}$ & A & $7,97 \pm 4,14$ a & A & $4,66 \pm 0,66 \mathrm{~b}$ & A & 0,56 & 43,3 & ** \\
\hline $\mathrm{Zn}(\mathrm{mg} \mathrm{kg}-1)$ & $1,51 \pm 0,82 \mathrm{~b}$ & B & $2,03 \pm 2,11 \mathrm{ab}$ & B & $1,16 \pm 0,34 \mathrm{~b}$ & B & $3,58 \pm 3,08 \mathrm{a}$ & A & $1,04 \pm 0,13 \mathrm{~b}$ & B & 0,49 & 84,95 & ** \\
\hline $\mathrm{Mn}(\operatorname{mg~kg-1)}$ & $1,52 \pm 0,25 \mathrm{a}$ & B & $2,76 \pm 19 \mathrm{a}$ & B & $2,17 \pm 0,13 \mathrm{a}$ & B & $3,03 \pm 2,71 \mathrm{a}$ & B & $1,88 \pm 0,16 \mathrm{a}$ & B & 0,45 & 90,11 & ns \\
\hline B (mg kg-1) & $0,41 \pm 0,25 \mathrm{ab}$ & A & $0,43 \pm 0,19 \mathrm{ab}$ & A & $0,29 \pm 0,13 \mathrm{~b}$ & M & $0,29 \pm 0,08 \mathrm{~b}$ & M & $0,53 \pm 0,16 \mathrm{ab}$ & A & 0,41 & 49,47 & * \\
\hline \multicolumn{14}{|c|}{ Relaciones Catiónicas } \\
\hline $\mathrm{Ca} / \mathrm{Mg}$ & $1,76 \pm 0,39 \mathrm{ab}$ & B & $1,57 \pm 0,58 \mathrm{ab}$ & B & $1,81 \pm 0,44 \mathrm{a}$ & B & $1,28 \pm 0,23 \mathrm{~b}$ & B & $1,76 \pm 0,31 \mathrm{a}$ & B & 0,46 & 23,83 & * \\
\hline $\mathrm{Mg} / \mathrm{K}$ & $12,349 \pm 6,42 \mathrm{a}$ & B & $25,08 \pm 30,48 \mathrm{a}$ & A & $25,79 \pm 8,98 \mathrm{a}$ & A & $15,05 \pm 8,09 \mathrm{a}$ & B & $14,06 \pm 6,41 \mathrm{a}$ & B & 0,41 & 74,19 & $\mathrm{~ns}$ \\
\hline $\mathrm{Ca} / \mathrm{K}$ & $20,58 \pm 310,21 \mathrm{~b}$ & B & $33,16 \pm 29,59 \mathrm{ab}$ & A & $46,88 \pm 21,38 \mathrm{a}$ & A & $18,73 \pm 9,22 \mathrm{~b}$ & B & $23,73 \pm 8,53 \mathrm{~b}$ & B & 0,58 & 55,29 & ** \\
\hline$(\mathrm{Ca}+\mathrm{Mg}) / \mathrm{K}$ & $32,93 \pm 16,26 \mathrm{ab}$ & B & $58,24 \pm 59,69 \mathrm{ab}$ & A & $72,67 \pm 29,36 \mathrm{a}$ & A & $33,78 \pm 17,09 \mathrm{~b}$ & B & $37,79 \pm 14,46 \mathrm{ab}$ & B & 0,51 & 60,77 & ** \\
\hline
\end{tabular}

N: neutro; LA: Ligeramente Acido; Ac: Acido; A: Alto; M: Medio; B: Bajo; NS: No Salino; ANOVA: análisis de varianza; Sig: significancia estadística; ns: no significativo $(p>0,05)$; * significativo $(p<0,05)$; ** altamente significativo $(p<0,001)$; R2: coeficiente de determinación; CV: coeficiente de variación. 
nicipios, evidenció que el $73 \%$ de los sitos analizados, el rango de variación del pH fue 6,0 a 7,3 (ligeramente ácido a neutro); el 12\% corresponde a suelos ácidos $(\mathrm{pH}<5,9)$; el $10 \%$, se evidenció en rango alcalino $(>7,3)$ y $5 \%$ restante presentaron condición acidez fuerte $(<5,5)$. Estos valores pueden estar asociados al material parental o formación (Combatt Caballero et al. 2008), lavado de bases cambiables ( $\mathrm{Ca}, \mathrm{Mg}, \mathrm{Na}$ y $\mathrm{K}$ ) y acumulación de cationes formado- res de ácido, como $\mathrm{Al}, \mathrm{Fe}$ y $\mathrm{Mn}$, que favorecen una mayor acidez (Sadeghian, 2016; Dutta et al. 2017; García Guzmán et al. 2019). En investigación realizada por Sadeghian (2016), encontró que la alternativa para corregir acidez del suelo se relacionaba con la aplicación de enmiendas agrícolas a base de carbonatos, óxidos o materiales silicatados (por ejemplo, $\mathrm{CaCO}_{3}$ o $\mathrm{MgCO}_{3}, \mathrm{CaO}, \mathrm{Ca}(\mathrm{OH})_{2}$ ), permitiendo la absorción de nutrientes por las plantas.
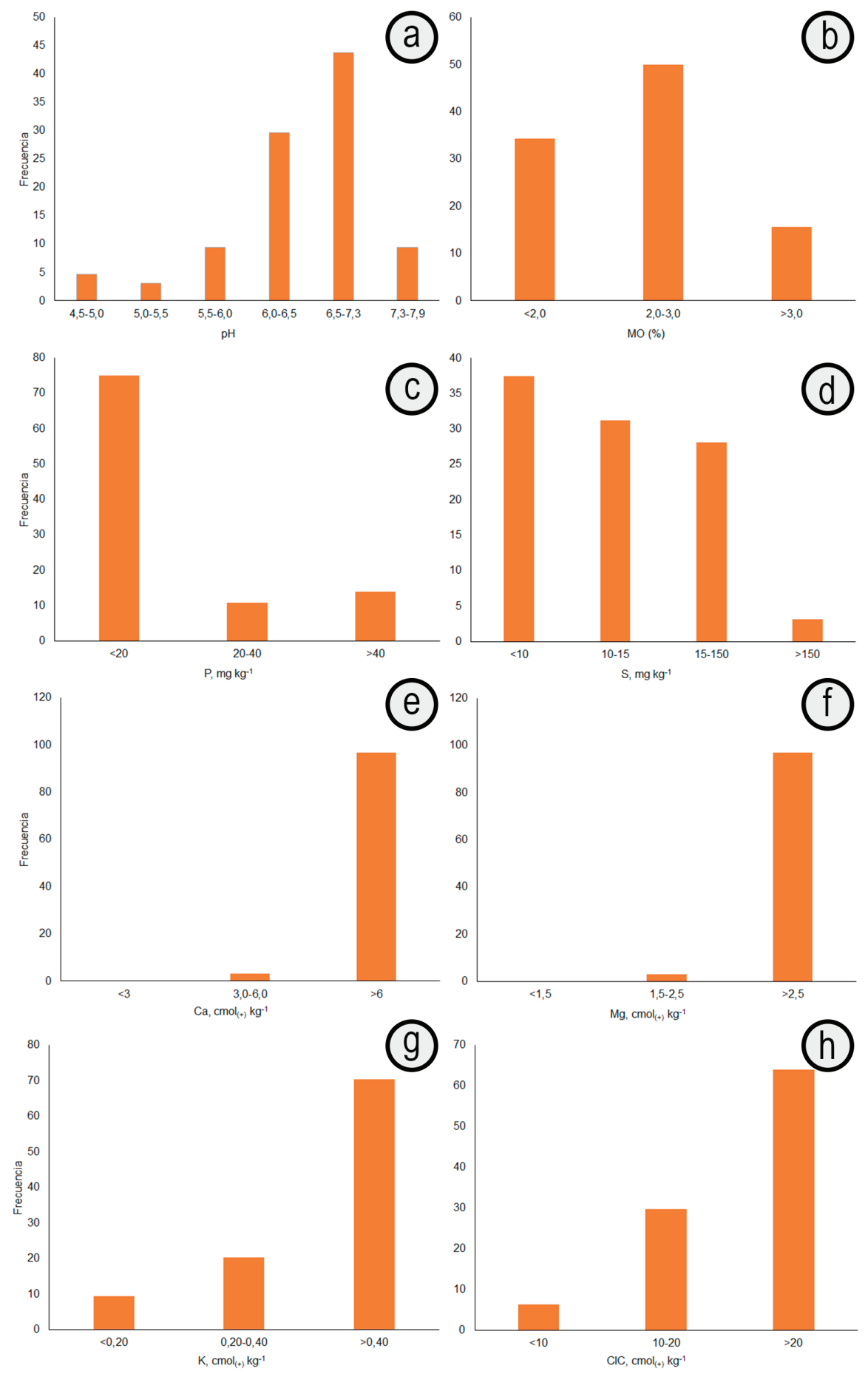

Figura 1. Distribución de frecuencia de propiedades químicas del suelo, a partir de 64 muestras de suelo cultivadas en maíz - algodón arroz, en la cuenca media y baja del río Sinú, Córdoba. a. pH; b. MO; c. P; d. S; e. Ca; f. Mg; g. K; h. CIC. 
El contenido de materia orgánica (MO) en los suelos dentro de los cultivos de maíz - algodón y arroz presentaron, que el 66\% de los sitios evaluados (42 sitios), valores superiores al 2,0\% (Figura 1B), con promedio de 2,39\%, indicando contenidos medios (ICA, 1992). El 34\% restante presentó contenidos bajos de MO (menor 2,0\%), siendo el municipio de Lorica donde se presentó el mayor número de sitios con valores superiores a 2,0\%, seguido de San Pelayo y San Carlos (Figura 1B); resultado similar reportan Martínez \& González (2017).

El fósforo $(\mathrm{P})$, se clasificó como bajo contenido en el suelo $(<20 \mathrm{mg}$ $\mathrm{kg}^{-1}$ ), en el $75 \%$ de los sitios analizadas, siendo Lorica, con prome-

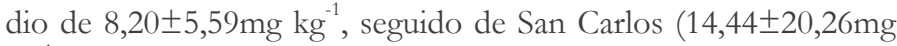
$\left.\mathrm{kg}^{-1}\right)$; los municipios con la mayor frecuencia de muestras, con valores en rango bajos. El 25\% de los sitios muestreados presentaron valores, que oscilan entre $21,73 \pm 17,39$ a $34,18 \pm 31,38 \mathrm{mg}$ $\mathrm{kg}^{-1}$ (medio), con promedio de 29,91 $\mathrm{mg} \mathrm{kg}^{-1}$ (Figura 1C). El azufre (S) mostró valores superiores a $15 \mathrm{mg} \mathrm{kg}^{-1}$, en más del $28 \%$ de las muestras analizadas y superiores a $150 \mathrm{mg} \mathrm{kg}^{-1}$, en el $3 \%$ del total de los sitios muestreados (Figura 1D). Valores altos de $\mathrm{S}$ en el suelo, se relaciona con zonas susceptibles a inundación (zonas cenagosas). Estos resultados obtenidos en esta investigación coinciden con los reportados por Combatt Caballero et al. (2008), en suelos del Valle del Sinú, asociados a áreas con acumulaciones de sulfuro de hierro o pirita $\left(\mathrm{FeS}_{2}\right)$, cuya mineralización aporta contenidos de $\mathrm{S}$, que influyen sobre el $\mathrm{pH}$ del suelo (procesos de óxido - reducción de la materia orgánica).

Respecto a las bases intercambiables (Ca, $\mathrm{Mg}, \mathrm{K}$ y $\mathrm{Na}$ ), solo el $\mathrm{Ca}$ intercambiable presentó comportamiento diferencial en el municipio de San Carlos $\left(9,43 \pm 4,34 \mathrm{cmol}_{(+)} \mathrm{kg}^{-1}\right)$, respecto a los otros mu-

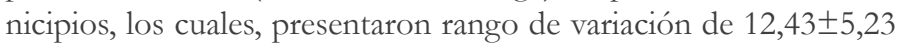
a $13,68 \pm 11,06 \mathrm{cmol}_{(+)} \mathrm{kg}^{-1}$. Igualmente, se evidenció que en más del $85 \%$ de los sitios evaluados, los contenidos de $\mathrm{Ca}, \mathrm{Mg}$ y $\mathrm{K}$ presentan contenidos medio a alto (ICA, 1992) (Figura 1E, F y G). Estudios realizados por Combatt et al. (2012) y Mercado et al. (2015), encontraron que los valores de las bases de intercambio en suelos, en la subregión Valle del Sinú, se relaciona al material de partida (arcillolita, lodolita y conglomerado), con lentes calcáreos; sin embargo, estos contenidos no se reflejan a nivel soluble, debido a la baja capacidad de mineralización y de solubilización hacia la fase líquida del suelo y contrastando con los valores de la reacción.

Los microelementos (Fe, Cu, Zn y B) mostraron comportamiento diferencial $(p<0,05)$ en los suelos de los municipios evaluados, mostrando valores medios a altos, sin problemas de deficiencias o toxicidades (Tabla 3).

La relación catiónicas $\mathrm{Ca} / \mathrm{Mg}$ mostró comportamiento diferencial $(p<0,05)$ en el suelo, en los municipios bajo los cultivos de maíz-algodón y arroz, mostrando que fue menor a 2, en todos los municipios, siendo más crítica esta relación en los municipios de San

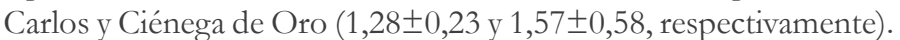
$\mathrm{La}$ relación $\mathrm{Mg} / \mathrm{K}(>20), \mathrm{Ca} / \mathrm{K}(>30)$ y $(\mathrm{Ca}+\mathrm{Mg}) / \mathrm{K}(>50)$ evidencian deficiencias de potasio $(\mathrm{K})$ en los municipios de Ciénaga de Oro y Lorica (Tabla 3). El principal problema que presentan los suelos dedicados a la producción de maíz, algodón y arroz, en el Valle del Sinú, se relaciona con la fracción soluble, en donde los contenidos de magnesio superan a los de calcio en todos los municipios, indicando una relación $\mathrm{Ca} / \mathrm{Mg}$-invertida (Tabla 3). Lo anterior, genera una condición indeseable en el suelo, debido a que el Mg, en abundancia, presenta características de desagregación, ahondando los problemas estructurales, que ocasionan limitantes para la absorción de otros elementos fundamentales para la planta, como son el Ca y K.

Es notoria la influencia del manejo agronómico del sistema productivo maíz, algodón y arroz, en cuanto a las características fisicoquímicas de suelo encontradas (altos valores de densidad aparente, contenido medio de materia orgánica, alta capacidad de intercambio catiónico), teniendo en cuenta que este sistema tecnificado sigue parámetros homogéneos, en cuanto a las prácticas de mecanización y de fertilización.

El análisis de componentes principales (ACP) permitió observar el comportamiento de los 25 indicadores fisicoquímicos de suelo evaluados, resaltando un conjunto mínimo de datos (CMD), que fueron capaz de explicar el comportamiento del suelo. Se encontró, que siete componentes principales (CP) obtuvieron valores propios $>1$, que explican el 81,56\% de la varianza total (Tabla 4). El CP1 (explica el 20,85\% de la varinaza), encontrando que este componente, estuvo dominado por la reacción del suelo $\mathrm{pH}(-0,84)$, seguido de los microelementos $\mathrm{Mn}(0,82), \mathrm{Fe}(0,81)$ y Cu $(0,79)$. El CP2 (explica el 19,22\% de la varianza) y este CP estuvo dominado por las relaciones catiónicas, siendo la relación $\mathrm{Mg} / \mathrm{K}$, la que presentó mayor valor absoluto $(0,96)$, seguida por $(\mathrm{Ca}+\mathrm{Mg}) / \mathrm{K}(0,95)$ y $\mathrm{Ca} / \mathrm{K}(0,90)$. El CP3 (explica el 16,04\% de la varianza) y este componente estuvo dominado por la base de intercambio $\mathrm{Mg}(0,95)$, seguido por la CIC $(0,91)$. Para el CP4 (explica el 8,83\% de la varianza), que estuvo dominado por la $\mathrm{CE}(0,96)$, seguido de $\mathrm{S}(0,89)$ y Na $(0,89)$. El CP5 (explica el 6,14\% de la varianza), dominado por los indicadores de suelo P $(0,82), \mathrm{K}(0,80)$ y DMP $(0,78)$. El CP6 (explica el 5,57\% de la varianza), dominado por los indicadores Da y Pt $(0,93)$ y, el último, CP7 (explica el 4,92\% de la varianza), siendo la Mip $(0,87)$, el indicador de suelo de mayor relevancia. Con el fin de obtener el CMD, se emplearon análisis de correlaciones lineales de Pearson $(p<0,05)$, obteniendo que los indicadores edáficos de mayor relevancia en el área de estudio fueron: la reacción del suelo $\mathrm{pH}$ (CP1), la relación catiónica $\mathrm{Mg} / \mathrm{K}$ (CP2), contenido de $\mathrm{Mg}$ (CP3), condición de salinidad del suelo CE (CP4), P (CP5) y las propiedades fisicas de suelo Da (CP6) y Mip (CP7). Siendo estas variables relevantes en el entendimiento de manejo o rehabilitación de los suelos, ubicados en el Valle del Sinú.

De los anteriores resultados, se concluye que, desde el punto de vista fisicoquímico de suelo, existe restricción para el desarrollo de las raíces de los cultivos (maíz - algodón y arroz), en los suelos de la subregión Valle del Sinú; sin embargo, las principales limitantes están relacionadas con las propiedades químicas del suelo, enfocadas a la reacción del suelo $(\mathrm{pH})$ y las relaciones catiónicas existentes. Se recomienda la implementación de prácticas de conservación de suelo, enfocadas en mejorar las propiedades fisicoquímicas del suelo. 
Tabla 4. Resultados análisis componentes principales de parámetros fisicoquimicos de suelo, bajo los sistemas agricolas maíz - algódon y arroz, Valle del Sinú.

\begin{tabular}{|c|c|c|c|c|c|c|c|c|}
\hline \multicolumn{9}{|c|}{ Componentes principales } \\
\hline & CP 1 & $\mathrm{CP} 2$ & CP 3 & $\mathrm{CP} 4$ & CP 5 & CP 6 & CP 7 & \\
\hline Autovalor & 5,21 & 4,80 & 4,01 & 2,21 & 1,53 & 1,39 & 1,23 & \\
\hline Varianza $(\%)$ & 20,85 & 19,22 & 16,04 & 8,83 & 6,14 & 5,57 & 4,92 & \\
\hline $\begin{array}{l}\text { Acumulada } \\
(\%)\end{array}$ & 20,85 & 40,07 & 56,11 & 64,94 & 71,07 & 76,64 & 81,56 & \\
\hline $\begin{array}{l}\text { Indicador de } \\
\text { Suelo++ }\end{array}$ & \multicolumn{7}{|c|}{ Vectores propios +} & Comunalidades \\
\hline $\mathbf{P t}$ & 0,23 & $-0,01$ & 0,12 & 0,07 & 0,04 & $\underline{0,93}$ & 0,03 & 0,95 \\
\hline $\mathrm{Mp}$ & 0,39 & 0,06 & $-0,06$ & 0,25 & $-0,12$ & 0,27 & $-0,68$ & 0,77 \\
\hline Msp & $-0,26$ & 0,06 & $-0,05$ & $-0,18$ & 0,10 & $-0,31$ & $-0,58$ & 0,54 \\
\hline Mip & $-0,02$ & $-0,19$ & 0,20 & $-0,20$ & 0,13 & 0,07 & $\underline{0,87}$ & 0,90 \\
\hline DMP & 0,02 & 0,03 & 0,14 & $-0,18$ & $\underline{0,78}$ & 0,06 & $-0,10$ & 0,67 \\
\hline $\mathrm{Da}$ & $-0,21$ & $-0,03$ & $-0,12$ & $-0,13$ & 0,06 & $-0,93$ & $-0,03$ & 0,95 \\
\hline $\mathrm{pH}$ & $-0,84$ & $-0,15$ & 0,10 & $-0,03$ & 0,23 & $-0,10$ & 0,08 & 0,81 \\
\hline $\mathrm{CE}$ & $-0,05$ & 0,04 & 0,11 & $\underline{0,96}$ & 0,00 & 0,05 & $-0,06$ & 0,94 \\
\hline M.O & 0,27 & $-0,09$ & 0,49 & $-0,01$ & 0,17 & 0,32 & 0,16 & 0,47 \\
\hline $\mathbf{P}$ & $-0,19$ & $-0,35$ & $-0,13$ & 0,04 & $\underline{0,82}$ & $-0,09$ & 0,12 & 0,87 \\
\hline $\mathrm{S}$ & 0,16 & 0,06 & 0,16 & $\underline{0,89}$ & $-0,05$ & 0,13 & $-0,01$ & 0,85 \\
\hline $\mathrm{Ca}$ & $-0,39$ & $-0,20$ & 0,77 & 0,19 & $-0,01$ & 0,08 & $-0,07$ & 0,84 \\
\hline $\mathrm{Mg}$ & $-0,01$ & 0,05 & $\underline{0,95}$ & 0,17 & $-0,03$ & 0,08 & 0,13 & 0,95 \\
\hline $\mathbf{K}$ & 0,02 & $-0,45$ & 0,02 & 0,00 & $\underline{0,80}$ & $-0,03$ & 0,13 & 0,86 \\
\hline $\mathrm{Na}$ & $-0,04$ & 0,13 & 0,24 & $\underline{0,89}$ & $-0,08$ & 0,04 & $-0,12$ & 0,88 \\
\hline CICE & $-0,15$ & $-0,15$ & $\underline{0,91}$ & 0,25 & 0,08 & 0,08 & 0,02 & 0,94 \\
\hline $\mathrm{Fe}$ & $\underline{0,81}$ & $-0,13$ & $-0,10$ & $-0,01$ & 0,14 & 0,28 & 0,05 & 0,79 \\
\hline Mn & $\underline{0,82}$ & 0,03 & $-0,06$ & $-0,01$ & 0,03 & 0,07 & $-0,04$ & 0,68 \\
\hline $\mathrm{Zn}$ & 0,63 & $-0,19$ & $-0,06$ & 0,06 & 0,61 & 0,13 & 0,08 & 0,85 \\
\hline $\mathrm{Cu}$ & $\underline{0,79}$ & $-0,31$ & 0,28 & 0,07 & $-0,07$ & 0,21 & 0,04 & 0,86 \\
\hline B & $-0,32$ & $-0,38$ & 0,22 & 0,08 & 0,32 & $-0,16$ & 0,12 & 0,44 \\
\hline $\mathrm{Ca} / \mathrm{Mg}$ & $-0,45$ & $-0,12$ & $-0,62$ & $-0,04$ & 0,07 & 0,07 & $-0,29$ & 0,70 \\
\hline $\mathrm{Mg} / \mathrm{K}$ & 0,00 & $\underline{0,96}$ & 0,10 & 0,07 & $-0,13$ & $-0,02$ & 0,00 & 0,96 \\
\hline $\mathrm{Ca} / \mathrm{K}$ & $-0,13$ & $\underline{0,90}$ & $-0,20$ & 0,10 & $-0,21$ & $-0,01$ & $-0,15$ & 0,94 \\
\hline$(\mathrm{Ca}+\mathrm{Mg}) / \mathrm{K}$ & $-0,08$ & $\underline{0,95}$ & $-0,08$ & 0,09 & $-0,18$ & $-0,01$ & $-0,09$ & 0,98 \\
\hline
\end{tabular}

+Los datos resaltados en negrita, fueron los seleccionados para la obtención del conjunto mínimo de datos (CMD); ++Las abreviaturas son las mismas que las utilizadas en las tablas 2 y 3.

Agradecimientos: La investigación, se realizó bajo el proyecto "Recomendaciones para el manejo de labranza en el sistema algodón-maíz, uso eficiente de agua en arroz y manejo de nutrientes en algodón, para el Valle cálido del Alto Magdalena y Valle medio del río Sinú" ejecutado por la Corporación Colombiana de Investigación Agropecuaria AGROSAVIA - C.I. Turipaná. Conflictos de intereses: El manuscrito fue preparado y revisado con la participación de todos los autores, quienes declaramos que no existe ningún conflicto de intereses que ponga en riesgo la validez de los resultados presentados. Financiación: Este estudio fue financiado por el Ministerio de Agricultura y Desarrollo Rural (MADR) y la Corporación Colombiana de Investigación Agropecuaria (AGROSAVIA), en el marco de la agenda quinquenal 2012-2017. 


\section{REFERENCIAS}

1. ABID, M.; LAL, R. 2009. Tillage and drainage impact on soil quality: II. Tensile strength of aggregates, moisture retention and water infiltration. Soil and Tillage Research. 103(2):364-372.

https://doi.org/10.1016/j.still.2008.11.004

2. AGUILAR-BAROJAS, S. 2005. Fórmulas para el cálculo de la muestra en investigaciones de salud. Salud en Tabasco. 11(1-2):333-338.

3. BURT, R. 2014. Soil survey field and laboratory methods manual. Soil Survey Investigations Report No. 51, Version 2. US Department of Agriculture, Natural Resources Conservation Service, WA, USA.

4. CASTRO REBOLLEDO, M.I.; MESTRE CARRILLO, G.I.; NÚÑEZ AVELLANEDA, L.A. 2018. Cambio climático y fertilización: efectos en la biota de un arroyo andino. En: Correa, A. (ed.), Agrociencias y cambio climático. $1^{\mathrm{a}}$ ed. Universidad de La Salle, Bogotá. p. 75-85. Disponible desde Internet en:

https: / / ciencia.lasalle.edu.co/cgi/viewcontent.cgi?article $=1040 \&$ context $=$ libros $\#$ page $=71 \quad$ (con acceso el 25/07/2020).

5. COMBATT CABALLERO, E.; MARTÍNEZ LARA, Z.; PALENCIA, M. 2008. Generación de acidez por oxidación de pirita en suelos sulfatados ácidos interiores de clima cálido. Temas Agrarios. 13(1):32-39. https://doi.org/10.21897/rta.v13i1.662

6. COMBATT, E.M.; NOVOA, Y.R.; BARRERA, V.J. 2012. Caracterización química de macroelementos en suelos cultivados con plátano (Musa AAB Simmonds) en el departamento de Córdoba. Colombia. Acta Agronómica. 61(2):166-176.

7. COMISIÓN ECONÓMICA PARA AMÉRICA LATINA Y EL CARIBE CEPAL, CEPAL. 2018. Segundo informe anual sobre el progreso y los desafíos regionales de la Agenda 2030 para el Desarrollo Sostenible en América Latina y el Caribe. Santiago: Naciones Unidadas, 184p.

8. DEPARTAMENTO ADMINISTRATIVO NACIONAL DE ESTADÍSTICA, DANE. 2015. Encuesta Nacional Agropecuaria. Departamento Administrativo Nacional de Estadística. Colombia. Disponible desde internet en: https://www.dane.gov.co/index.php/estadisticas-porte$\mathrm{ma} /$ agropecuario/encuesta-nacional-agropecuaria-ena (con acceso 01/08/2019).

9. DUIKER, S.W.; RHOTON, F.E.; TORRENT, J.; SMECK, N.E.; LAL, R. 2003. Iron (Hydr) Oxide Crystallinity
Effects on Soil Aggregation. Soil Science Society of America J. 67(2):606-611.

https://doi.org/10.2136/sssaj2003.6060

10. DUTTA, M.; PHOM, B.; RAM, S. 2017. Physico-chemical properties of soils under different land uses in Longleng district soils of Nagaland. An Asian J. Soil Science. 12(2):307-313.

https://doi.org/10.15740/has/ajss/12.2/307-313

11. FEDERACIÓN NACIONAL DE CULTIVADORES DE CEREALES Y LEGUMINOSAS, FENALCE. 2019. Indicadores Cerealistas 2019-A. Departamento Económico y Apoyo a la Comercialización. 72p. Disponible desde Internet en:

https://www.fenalce.org/archivos/indicerealista2019A. pdf (con acceso 28/10/2019).

12. FU, Y.; TIAN, Z.; AMOOZEGAR, A.; HEITMAN, J. 2019. Measuring dynamic changes of soil porosity during compaction. Soil and Tillage Res. 193:114-121. https://doi.org/10.1016/j.still.2019.05.016

13. GARCÍA GUZMÁN，S.; BAUTISTA-MONTEALEGRE, L.; BOLAÑOS-BENAVIDES, M. 2019. Diagnóstico de la fertilidad de los suelos de cuatro municipios de Cundinamarca (Colombia) para la producción de plátano. Rev. U.D.C.A Act. \& Div. Cient. 22(1):e1192. https://doi.org/10.31910/rudca.v22.n1.2019.1192

14. GÓMEZ, K.A.; GÓMEZ, A.A. 1984. Statistical procedures for agricultural research. 2nd Ed. John Wiley \& Sons, INC., UK. 657p.

15. HERNÁNDEZ JIMÉNEZ, A.; VERA MACÍAS, L.; NAVEDA BASURTO, C.A.; GUZMÁN CEDEÑO, A.M.; VIVAR ARRIETA, M.R.; ZAMBRANO, TEÓDULO, M.G.; FREDDY ORMANZA, K.; LEÓN AGUILAR, R.V.; LÓPEZ ALAVA, G.A. 2017. Variaciones en algunas propiedades del suelo por el cambio de uso de la tierra, en las partes media y baja de la microcuenca Membrillo, Manabí, Ecuador. Cultivos Tropicales. 38(1):50-56.

16. HOLDRIDGE, L. 2000. Ecología basada en zonas de vida. Instituto Interamericano de Cooperación para la Agricultura, IICA. San José de Costa Rica. 225p.

17. INSTITUTO COLOMBIANO AGROPECUARIO, ICA. 1992. Fertilización en diversos cultivos. Quinta aproximación. Manual de Asistencia Técnica No. 25. Instituto Colombiano Agropecuario. (Colombia). 64p.

18. INSTITUTO DE HIDROLOGÍA, METEOROLOGÍA Y ESTUDIOS AMBIENTALES, IDEAM; U.D.C.A. 2015. Síntesis del estudio nacional de la degradación de suelos por erosión en Colombia - 2015. IDEAM - MADS. Bo- 
gotá D.C., Colombia., 62p. Publicación aprobada por el IDEAM, diciembre de 2015, Bogotá D.C., Colombia.

19. INSTITUTO GEOGRÁFICO AGUSTÍN CODAZZI, IGAC. 2006. Métodos analíticos del laboratorio de suelos, sexta edición. Imprenta Nacional de Colombia. Bogotá. 648p.

20. INSTITUTO GEOGRÁFICO AGUSTÍN CODAZZI, IGAC. 2009. Subdirección Agrológica. Estudio general de suelos y zonificación de tierras del Departamento de Córdoba. Bogotá - Colombia. 502p.

21. INSTITUTO GEOGRÁFICO AGUSTÍN CODAZZI, IGAC. 2017. Etapa de campo para levantamiento de suelos. Subdirección Agrológica. 27p. Disponible desde Internet en:

https://www.igac.gov.co/sites/igac.gov.co/files/i401001016.v1 etapadeposcampoparaloslevantamientosdesuelos. pdf (con acceso el 14/05/2018).

22. MÁRQUEZ COVARRUBIAS, H. 2009. Diez rostros de la crisis civilizatoria del sistema capitalista mundial. Problemas del desarrollo. 40(159):191-210.

23. MARTÍNEZ, Z.; GONZÁLEZ, M. 2017. Contaminación de suelos agrícolas por metales pesados, zona minera $\mathrm{El} \mathrm{Ala-}$ crán, Colombia. Temas Agrarios. 22(2):21-31.

https://doi.org/10.21897/rta.v22i2.941
24. MERCADO, J.L.; COMBATT, E.M.; PALENCIA, M.S. 2015. Adición de polielectrolitos sobre la fase acuosa de un suelo sulfatado ácido interior de Córdoba, Colombia. Temas Agrarios. 20(1):60-70.

https://doi.org/10.21897/rta.v20i1.748

25. NAVARRO BRAVO, A.; FIGUEROA SANDOVAL, B.; SANGERMAN J.; DORA, M.A.; OSUNA CEJA, E.S. 2012. Propiedades físicas y químicas del suelo bajo labranza de conservación y su relación con el rendimiento de tres cultivos. Rev. Mex. Ciencias Agrícolas. 3(spe4):690697.

26. PRIETO, B.; PEROZA, J.; GRANDET, G. 2010. Efecto de labranza y manejo de materiales orgánicos sobre algunas propiedades físicas y químicas de un vertic endoaquept del valle del Sinú, Córdoba Colombia. Temas Agrarios. 15(2):27.

https://doi.org/10.21897/rta.v15i2.677

27. SADEGHIAN, S. 2016. La acidez del suelo una limitante común para la producción de café. Avances técnicos. Programa de investigación científica. Fondo nacional del café. Cenicafé. (Colombia). 12p.

28. UNITED STATES DEPARTMENT OF AGRICULTURE, USDA. 2014. Claves para la taxonomía de suelos. 12a ed. USDA, WA, USA. 